Susana J. Gotthelf ${ }^{1}$

Patricia C. Rivas ${ }^{2}$

${ }^{1}$ Especialista y Magister en Salud Pública.

Médica adjunta del

Departamento de

Investigación Epidemiológica

Centro Nacional

de Investigaciones

Nutricionales. Salta

Administración Nacional de

Laboratorios e Institutos de

Salud. ANLIS.

${ }^{2}$ Magister en Ciencias de la

Nutrición.

Bioquímica adjunta

del Departamento de

Investigación Epidemiológica

Centro Nacional

de Investigaciones

Nutricionales. Salta Administración Nacional de Laboratorios e Institutos de Salud. ANLIS.

Trabajo recibido: 15 de Noviembre 2018.

Aprobado: 19 de Marzo

\title{
SÍNDROME METABOLICO Y OBESIDAD SEGÚN CRITERIOS IDF/ALAD EN ADULTOS DE LA CIUDAD DE SALTA
}

\section{METABOLIC SYNDROME AND OBESITY AC- CORDING TO IFD/LAAD CRITERIA IN ADULTS IN THE CITY OF SALTA}

SÍNDROME E OBESIDADE METABÓLICAS DE ACORDO COM OS CRITÉRIOS IDF I ALAD EM ADULTOS DA CIDADE DE SALTA

\section{Resumen}

Objetivo: Estimar prevalencia de Síndrome Metabólico en adultos de Salta según criterios de la Federación Internacional de Diabetes (IDF) y Asociación Latinoamericana de Diabetes (ALAD). Analizar relación entre obesidad y Síndrome Metabólico.

Metodología: estudio transversal, base de datos secundaria (Encuesta Nutricional de Salta, 2014).Variables: SM: criterios IDF/ALAD, sexo, edad, estado nutricional .Análisis: distribución de frecuencias,comparación de medias .Regresión logística. EXCEL, SPSS 18.

Resultados: Se evaluaron 425 adultos, 63,5\% mujeres. La prevalencia de SM fue 20,9\% según ALAD, 30,8\% según IDF, mayor en varones (34,2 y 41,9\%).La prevalencia de Sobrepeso/Obesidadfue 61,8\%.Los valores medios de IMC, triglicéridos y presión arterial en varones superaron los límites críticos.Los modelos de regresión (IDF/ALAD) mostraron que la probabilidad de desarrollar SM fue mayor en varones, aumentó con la edad ( $\geq 65$ años) y con sobrepeso/ obesidad.

Conclusiones: se observó diferencias en las prevalencias de SM según criterio diagnóstico utilizado, asociándose al sobrepeso/obesidad.

Palabras Clave: Sindrome Metabólico, obesidad, adultos. 


\section{Abstract:}

Objective: To estimate the prevalence of Metabolic Syndrome in adults in Salta according to criteria from International Federation of Diabetes (IFD) and Latin American Association of Diabetes (LAAD). Analyze the relationship between obesity and Metabolic Syndrome. Methodology: cross-sectional study, secondary data base (Nutritional Survey, Salta. 2014) Variables: MS: criteria IFD/LAAD, sex, age, nutritional status. Analysis: frequency distribution, comparison of measures. Logistic regression. EXCEL, SPSS 18.

Results: We evaluated 425 adults; $63.5 \%$ women. The prevalence of MS was $20.9 \%$ according to LAAS, $30.8 \%$ according to IFD, greater in males (34.2 and $41.9 \%$ ). The prevalence of Overweight/Obesity was $61.8 \%$. The mean values of BMI, triglycerides and blood pressure in males were higher than the critical limits. Regression models (IFD/ LAAD) showed that the probability to develop MS was greater in males, increased with age ( $\geq 65$ years) and with overweight/obesity.

Conclusions: we observed differences in the prevalence of MS according to the medical diagnosis used, being associated with overweight/obesity.

Key Words: Metabolic síndrome, Obesity, adults.

Resumo:

Objetivo: estimar a prevalência de síndrome metabólica em adultos de Salta de acordo com os critérios da Federação Internacional de Diabetes (IDF) e Latin American Diabetes Association (ALAD). Analisar a relação entre obesidade e síndrome metabólica. Metodologia: Transversal, banco de dados secundária (Enquete Nutricional de Salta, 2014) .Variàveis: SM: criterios IDF / ALAD, sexo, idade, estado nutricional . Análise: distribuição de freqüência significa comparação de médias. Regressão logística. EXCEL, SPSS 18. Resultados: Foram avaliados 425 adultos, deles, 63,5\% foram mulheres. A prevalência de de Excesso de Peso/Obesidade foi de 20,9\% segundo ALAD, 30,8\% de acordo com IDF, maior em homens (34,2 e 41,9\%). A prevalência de Excesso de Peso / Obesidad foi $61,8 \%$. Os valores médios de IMC, triglicéridos e pressão arterial em homens excederam os limites críticos. Os modelos de regressão (IDF / ALAD) evidenciaram que a probabilidade de desenvolver SM era maior em homens, aumentou com a idade ( $\geq 65$ anos ou mais) e com excesso de peso / obesidade.

Conclusões: foram observadas diferenças na prevalência de SM segundo o critério diagnóstico utilizado, estando associadas ao sobrepeso / obesidade.

Palavras chave: Sindrome metabolico, obesidade, adultos.

\section{Introducción}

La obesidad (O) y el síndrome metabólico (SM) son entidades clínicas complejas y heterogéneas con fuerte componente genético, cuya expresión está influenciada por factores ambientales, sociales, culturales y económicos. En la fisiopatogenia del SM, la obesidad parece ser uno de los factores desencadenantes más importantes entre otras alteraciones metabólicas que lo caracterizan: intolerancia a la glucosa, diabetes, dislipidemia e hipertensión .

El incremento paralelo de la prevalencia de la obesidad y del SM es un fenómeno mundial y Argentina no es la excepción. Ambas patologías son factores de riesgo importantes para el desarrollo de diabetes tipo 2, enfermedad arterial coronaria y cerebrovascular por arteriosclerosis, que son las principales causas de muerte en nuestro país. La alta prevalencia de la obesidad es una realidad en los países industrializados y en los que están en vías de desarrollo. La información disponible indica un aumento acelerado del problema que de no contenerse, puede tener repercusiones importantes en los indicadores 
de salud de muchos países incluyendo el nuestro1. En nuestro país los datos de la Tercera Encuesta Nacional de Factores de Riesgo , muestran que entre el 2005 al 2013, la prevalencia de obesidad aumentó un 42,5\%. La Región del Noroeste Argentino, a la cual pertenece la provincia de Salta, muestra los más bajos indicadores de desarrollo humano que se articulan con una cada vez más creciente evolución hacia la obesidad.

El SM se caracteriza por la aparición en forma simultánea o secuencial de diversas alteraciones metabólicas, e inflamatorias a nivel molecular, celular o hemodinámico asociadas a la presencia de resistencia a la insulina y de adiposidad de predominio visceral. Existen diferentes criterios diagnósticos para SM, las definiciones más utilizadas son las de la Federación Internacional de Diabetes (IDF) y del American HeartAssociation, Nationalcolesterol EducationProgramAdultTreatment Panel III (ATP III). La IDF usa la circunferencia de cintura (CC) como referente importante. La CC es uno de los mejores indicadores antropométricos de carácter predictivo de enfermedad cardiovascular, diferentes estudios permiten inferir que los incrementos en función del tiempo repercuten en un aumento del SM. En el 2009 se realizó un consenso armonizado según varias entidades (IDF/NHLBI/AHA-2009), en un intento para unificar criterios acordándose que no debía haber ningún componente mandatorio, y quepara la población latinoamericana se usarían los mismos puntos de corte de CC del sudeste asiático. Así mismo se reconoció la necesidad de ajustar los parámetros para el diagnóstico de obesidad abdominal a las características étnicas y regionales.

Recientemente, la Asociación Latinoamericana de Diabetes - ALAD ha publicado sus criterios diagnósticos, en base a la definición de la IDF, especificando las medidas que se debe utilizar para evaluar perímetro abdominal en la Región de América Latina. Recomienda utilizar en la práctica clínica la definición de la IDF con nuevos puntos de corte de CC para población latinoamericana: $94 \mathrm{~cm}$ en hombres y $88 \mathrm{~cm}$ en mujeres .

\section{Objetivo General}

- $\quad$ Estimar la prevalencia de SM y sus componentes en adultos de la ciudad de Salta según criterios IDF y ALAD y analizar la relación entre obesidad y SM.

\section{Materiales y Métodos}

Los datos fueron extraídos de la base de la Encuesta Nutricional de Salta Capital (2014),llevada a cabo por el Centro Nacional de InvestigacionesNutricionales (CNIN).

Estudio transversal, estratificado, bietápico(fracciones/radios censales y hogares). Población: adultos de ambos sexos pertenecientes a hogares de la ciudad de Salta evaluados entre abril y junio de 2014 .

\section{Variables:}

- Sexo, edad.

- SM: criterios IDF : Circunferencia de Cintura (CC) mujeres $\geq 80 \mathrm{~cm}$, varones $\geq 90 \mathrm{~cm}$ más dos de las siguientes variables:Triglicéridos(TG) $\geq 150 \mathrm{mg} / \mathrm{dl}$; Colesterol HDL (HDL): mujeres $\leq 50 \mathrm{mg} / \mathrm{dl}$, varones $\leq 40 \mathrm{mg} / \mathrm{dl}$; Presión sistólica/diastólica (PAS/ PAD) $\geq 130 / 85 \mathrm{mmHg}$; Glucemia basal (GB) $\geq 100 \mathrm{mg} / \mathrm{dl}$.

Criterios ALAD3:CC mujeres $\geq 88 \mathrm{~cm}$, varones $\geq 94 \mathrm{~cm}$ más dos de las siguientes variables $\mathrm{TG} \geq 150 \mathrm{mg} / \mathrm{dl}$; HDL: mujeres $\leq 50 \mathrm{mg} / \mathrm{dl}$, varones $\leq 40 \mathrm{mg} / \mathrm{dl} ; \mathrm{PAS} / \mathrm{PAD}$ $\geq 130 / 85 \mathrm{mmHg}$; Glucemia basal $\geq 100 \mathrm{mg} / \mathrm{dl}$.

- Estado nutricional según Índice de Masa Corporal (IMC):Normal <25; Sobrepeso $\geq 25$ y $<30$; Obesidad $\geq 30$. Clasificación Organización Mundial de Salud (OMS) .

- Mediciones antropométricas:Peso corporal: balanza de pie o plataforma tipo CAM, capacidad $150 \mathrm{~kg}$. Se pesó con mínimo de ropas, registrándose el peso completo en 
kilogramosy gramos.

Longitud corporal: posición de pie, cinta métrica metálica graduada encm y mm.Se midió sin calzado ni objetos en la cabeza, luego de realizaruna inspiración profunda, se registró la medida en $\mathrm{cm}$ y $\mathrm{mm}$.

CC: cinta métrica flexible, inextensible, milimetrada, ancho no mayora $5 \mathrm{~mm}$. Procedimiento: paciente de pie, cinta alrededor del abdomen, $1 \mathrm{~cm}$ aproximadamente porarriba de las crestas iliacas. La lectura se realizó a la alturadel ombligo.

Presión arterial:tensiómetro digital (Microlife BP 3BTO-A) método de medición oscilométrico.Se usaron brazaletes para adultos y obesos. Se promediaron dos tomas de presión con intervalo de 15 minutos.

- Parámetros bioquímicos: Colesteroltotal (COL), HDL y TG. determinados a partir de sangre periférica,mediante metodología enzimática colorimétrica, bajo control de calidad interno y externo PEEC (Programa de Evaluación Externa de Calidad, Fundación Bioquímica Argentina).Criterios de exclusión: incumplimiento horas de ayuno,falta de consentimiento.

Análisis estadístico: Los resultados se presentan en tablas y gráficos de distribución de frecuencias y de asociación Chi2, Fisher. Kolgomorov (normalidad),Comparación de valores medios U Mann-Whitney,Test de Student. Se consideró un $\mathrm{p}<0,05$ como punto de cortepara la significancia estadística. Programas estadísticos: EXCEL, SPSS 18.

Consideraciones éticas:Todos los individuos firmaron un consentimiento informado previo al estudio, adhiriendo a las normativas internacionales vigentesde acuerdo a la declaración de Helsinki.

\section{Resultados}

Se evaluaron 425 adultos (20-88 años) de ambos sexos,con prevalencia de mujeres $(63,5 \%)$. La edad promedio de la muestra fue 41,12 años $\pm 15,18$. La prevalencia global de SM encontrada fue de 20,9\% según criterio ALAD y 30,8\% según IDF, con diferencia estadísticamente significativa según sexo, siendo más frecuente en varones (Tabla 1). La distribución de la muestra según magnitud delsobrepeso fue la siguiente: 29,6\% con sobrepeso y $32,2 \%$ con obesidad.Los valores medios de IMC, en ambos sexos, se ubicaron en niveles de Sobrepeso. Los valores medios de TG y PAS en varones, se encontraron por encima de valores críticos.

Las prevalencias aisladas para cada uno de los factores de riesgo asociado al SM se describen en Tabla 2. La presencia de una CC aumentada fue el atributo más frecuente para el diagnóstico de SM en la población salteña, mostrando variaciones importantes según criterio (ALAD 30.7\% vs IDF 58.6\%) seguido de la hipertrigliceridemia (35,9\%). Se observaron diferencias significativas según género, siendo los hombres los que reportaron mayor prevalencia de hipertensión, hipertrigliceridemia y niveles de colesterol HDL bajos.

La prevalencia del SM estuvo directa y significativamenteasociada a la edad ( p 0,000), mostrando un incremento progresivo para ambos criterios: IDF $(9,5 \%$ a $29,5 \%)$, ALAD $(12.5 \%$ a $54 \%)$ (Gráfico 1$)$.

La prevalencia de SM estuvo directamente asociada a la magnitud y distribución del $\mathrm{S} / \mathrm{O}$, observándose un incremento notablesegún el $\mathrm{S} / \mathrm{O}$ se acompañe además de una $\mathrm{CC}$ aumentada, tanto en mujeres como varones (Tablas 3 y 4 ).

Los modelos de regresión (IDF/ALAD) muestran que la probabilidad de desarrollar SM es mayor en los varones, se incrementa con la edad,con el S/O y con la CC aumentada (Tabla 5).El riesgo de presentar un SM fue6,97 vecesmayor en individuos obesos y 19,35 en aquellos que además se acompañó de una $\mathrm{CC}$ aumentada. 
Tabla N¹: Características generales de la muestra según sexo. Salta.CNIN. 2014.

\begin{tabular}{|c|c|c|c|c|c|c|c|}
\hline & \multicolumn{2}{|c|}{ Mujeres (n: 270) } & \multicolumn{2}{|c|}{ Varones (n:155) } & \multicolumn{2}{|c|}{ Total (n: 425) } & \multirow[t]{2}{*}{ P valor } \\
\hline & $n$ & $\%$ & $n$ & $\%$ & $n$ & $\%$ & \\
\hline \multicolumn{8}{|c|}{ SINDROME METABOLICO } \\
\hline \multicolumn{8}{|l|}{ ALAD } \\
\hline No & 234 & 86,7 & 102 & 65,8 & 336 & 79,1 & \multirow{2}{*}{0,000} \\
\hline $\mathrm{Si}$ & 36 & 13,3 & 53 & 34,2 & 89 & 20,9 & \\
\hline \multicolumn{8}{|l|}{ IDF } \\
\hline No & 204 & 75,6 & 90 & 58,1 & 294 & 69,2 & \multirow{2}{*}{0,000} \\
\hline $\mathrm{Si}$ & 66 & 24,4 & 65 & 41,9 & 131 & 30,8 & \\
\hline \multicolumn{8}{|c|}{ ESTADO NUTRICIONAL } \\
\hline Normal & 104 & 38,5 & 58 & 37,4 & 162 & 38,2 & \multirow{3}{*}{0,91} \\
\hline Sobrepeso & 81 & 30 & 45 & 29 & 126 & 29,6 & \\
\hline Obesidad & 85 & 31,5 & 58 & 33,6 & 137 & 32,2 & \\
\hline
\end{tabular}

\begin{tabular}{lccccccc} 
VALORES MEDIOS Y DS & & & & & & & \\
Edad (años) & 38,09 & $\pm 15,48$ & 38,44 & $\pm 17,30$ & 38,22 & $\pm 16,18$ & 0,81 \\
IMC & 27,92 & $\pm 6,91$ & 28,13 & $\pm 6,39$ & 27,99 & $\pm 6,72$ & 0,76 \\
CC (cm) & 86,62 & $\pm 14,22$ & 93,78 & $\pm 15,38$ & 89,23 & $\pm 15,04$ & 0,000 \\
PAS (mm/Hg) & 120,77 & $\pm 18,14$ & 132,49 & $\pm 19,43$ & 125,1 & $\pm 19,44$ & 0,000 \\
PAD (mm/Hg) & 70,05 & $\pm 10,48$ & 76,59 & $\pm 12,23$ & 72,43 & $\pm 11,57$ & 0,000 \\
TG (mg/dl) & 138,96 & $\pm 75,30$ & 182,65 & $\pm 101,81$ & 154,9 & $\pm 88,34$ & 0,000 \\
HDL (mg/dl) & 49,25 & $\pm 13,11$ & 43,86 & $\pm 13,14$ & 47,29 & $\pm 13,36$ & 0,000 \\
GB (mg/dl) & 90,68 & $\pm 22,05$ & 93,77 & $\pm 28,69$ & 91,81 & $\pm 24,69$ & 0,21 \\
\hline
\end{tabular}

SM: síndrome metabólico. DS: Desvío estándar. CC: circunferencia de cintura. PAS/ PAD: presión arterial sistólica/diastólica. TG: triglicéridos. HDL: Colesterol HDL. GB: Glucemia basal. Chi cuadrado. T de Student y U Mann-Whitney . p $<0,05$ 
Tabla N²: Prevalencia de factores de riesgo asociados al Síndrome Metabólico según género. Salta.CNIN. 2014.

\begin{tabular}{lccccccc}
\hline & Mujeres (n: 270) & \multicolumn{2}{c}{ Varones (n:155) } & \multicolumn{1}{c}{ Total (n: 425) } & P valor \\
\hline PAS & $n$ & $\%$ & $n$ & $\%$ & $n$ & $\%$ & \\
Normal & 239 & 79,7 & 102 & 55,1 & 341 & 70,3 & 0,000 \\
Elevada & 61 & 20,3 & 83 & 44,9 & 144 & 29,7 & \\
& & & & & & & \\
PAD & & & & & & & \\
Normal & 278 & 92,7 & 148 & 80 & 426 & 87,8 & 0,000 \\
Elevada & 22 & 7,3 & 37 & 20 & 59 & 12,2 & \\
& & & & & & & \\
CC IDF & 113 & 37,7 & 88 & 47,6 & 201 & 41,4 & 0,032 \\
Normal & 187 & 62,3 & 97 & 52,4 & 284 & 58,6 &
\end{tabular}

\section{ALAD}

$\begin{array}{lccccccc}\text { Normal } & 225 & 75 & 111 & 60 & 336 & 69,3 & 0,010 \\ \text { Aumentada } & 75 & 25 & 74 & 40 & 149 & 30,7 & \end{array}$

Aumentada

\section{GB}

Normal

Aumentada

267

267

89
11

161

24

87
13

$\begin{array}{ccc}428 & 82,2 & 0,510 \\ 57 & 11,8 & \end{array}$

\section{HDL}

Normal

237

79

111

60

348

$\begin{aligned} & 71,8 \\ & 28,2\end{aligned} \quad 0,000$

Disminuido

63

21

74

40

137

28,2

$\begin{array}{lccccccc}\text { TG } & & & & & & & \\ \text { Normal } & 210 & 70 & 101 & 54,6 & 311 & 64,1 & \\ \text { Aumentado } & 90 & 30 & 84 & 45,4 & 174 & 35,9 & 0,001\end{array}$

SM: síndrome metabólico. CC: circunferencia de cintura. PAS/PAD: presión arterial sistólica/diastólica.

TG: triglicéridos. HDL: Colesterol HDL. GB: Glucemia basal. Chi cuadrado p<0,05.

\section{Gráfico 1: Prevalencia de SM según criterios ALAD/IDF por grupos estareos. CNIN. Salta. 2014.}

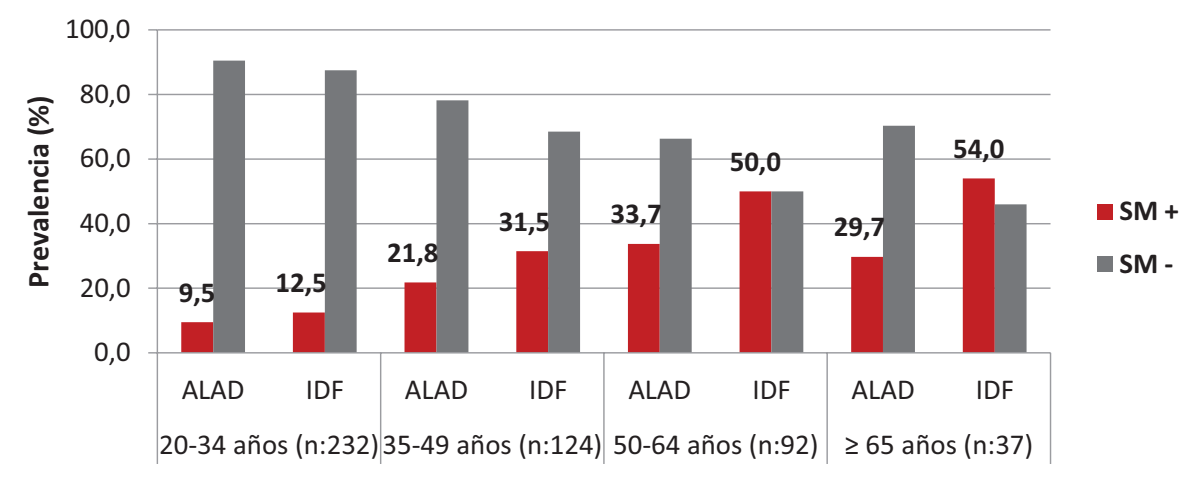


Tabla No3: Prevalencia de Síndrome metabólico según criterio ALAD en relación al estado nutricional, CC y género, en adultos de la ciudad de Salta. CNIN.2014.

\begin{tabular}{|c|c|c|c|c|c|c|}
\hline \multirow[b]{2}{*}{ Estado nutricional } & \multicolumn{2}{|c|}{ SM ALAD (-) } & \multicolumn{2}{|c|}{ SM ALAD (+) } & \multicolumn{2}{|c|}{ TOTAL } \\
\hline & & & $\mathrm{Mu}$ & & & \\
\hline & $n$ & $\%$ & $n$ & $\%$ & $n$ & $\%$ \\
\hline N/CC normal & 104 & 100 & 0 & 0 & 104 & 38,5 \\
\hline $\mathrm{S} / \mathrm{CC}$ normal & 51 & 100 & 0 & 0 & 51 & 18,9 \\
\hline $\mathrm{S} / \mathrm{CC}$ aumentada & 27 & 90 & 3 & 10 & 30 & 11,1 \\
\hline $\mathrm{O} / \mathrm{CC}$ normal & 8 & 100 & 0 & 0 & 8 & 3 \\
\hline $\mathrm{O} / \mathrm{CC}$ aumentada & 44 & 57,1 & 33 & 42,9 & 77 & 28,5 \\
\hline Total & 234 & 86,7 & 36 & 13,3 & 270 & 100 \\
\hline $\mathrm{P}$ valor & & & & & & \\
\hline
\end{tabular}

$\begin{array}{lcccccc}\text { Estado nutricional } & n & \% & n & \% & n & \% \\ \text { N/CC normal } & 56 & 96,6 & 2 & 3,4 & 58 & 37,4 \\ \text { S/CC normal } & 30 & 69,8 & 13 & 30,2 & 43 & 27,7 \\ \text { S/CC aumentada } & 0 & 0 & 2 & 100 & 2 & 1,3 \\ \text { O/CC normal } & 6 & 54,5 & 5 & 45,5 & 11 & 7,1 \\ \text { O/CC aumentada } & 10 & 24,4 & 31 & 75,6 & 41 & 26,5 \\ \text { Total } & 102 & 65,8 & 53 & 34,2 & 155 & 100 \\ \text { P valor } & & & 0,000 & & & \end{array}$

SM: síndrome metabólico. N: normal. S: sobrepeso. O: obesidad. CC: circunferencia de cintura. Chi cuadrado $\mathrm{p}<0,05$. 
Tabla N4: Prevalencia de Síndrome metabólico según criterio IDF en relación al estado nutricional, CC y género, en adultos de la ciudad de Salta. CNIN.2014.

\begin{tabular}{lcccccc}
\hline & \multicolumn{2}{c}{ SM IDF (-) } & \multicolumn{2}{c}{ SM IDF } & $(+)$ & \multicolumn{2}{c}{ TOTAL } \\
\hline Estado nutricional & \multicolumn{7}{c}{ Mujeres } \\
\hline N/CC normal & 97 & 93,3 & 7 & 6,7 & 104 & 38,5 \\
S/CC normal & 41 & 80,4 & 10 & 19,6 & 51 & 18,9 \\
S/CC aumentada & 20 & 66,7 & 10 & 33,3 & 30 & 11,1 \\
O/CC normal & 7 & 87,5 & 1 & 12,5 & 8 & 3 \\
O/CC aumentada & 39 & 50,6 & 38 & 49,4 & 77 & 28,5 \\
Total & 204 & 75,6 & 66 & 24,4 & 270 & 100 \\
P valor & & & 0,000 & & &
\end{tabular}

Estado nutricional

Varones

\begin{tabular}{lcccccc}
\hline & $n$ & $\%$ & $n$ & $\%$ & $n$ & $\%$ \\
N/CC normal & 52 & 89,7 & 6 & 10,3 & 58 & 37,4 \\
S/CC normal & 23 & 53,5 & 20 & 46,5 & 43 & 27,7 \\
S/CC aumentada & 0 & 0 & 2 & 100 & 2 & 1,3 \\
O/CC normal & 5 & 45,5 & 6 & 54,5 & 11 & 7,1 \\
O/CC aumentada & 10 & 24,4 & 31 & 75,6 & 41 & 26,5 \\
Total & 90 & 58,1 & 65 & 41,9 & 155 & 100 \\
P valor & & 0,000 & & & \\
\hline
\end{tabular}

SM: síndrome metabólico. N: normal. S: sobrepeso. O: obesidad. CC: circunferencia de cintura. Chi cuadrado $\mathrm{p}<0,05$. 
Tabla No5: Modelo de regresión logística para Síndrome Metabólico según relación Estado nutricional/CC ajustado por sexo y edad.

\begin{tabular}{|c|c|c|c|}
\hline Síndrome Metabólico & OR & IC $95 \%$ & P valor \\
\hline \multicolumn{4}{|l|}{ IDF } \\
\hline & \multicolumn{3}{|c|}{ Estado nutricional/CC } \\
\hline N/CC normal & 1 & & \\
\hline S/CC normal & 5,35 & $2,49-11,47$ & 0,000 \\
\hline $\mathrm{S} / \mathrm{CC}$ aumentada & 9,44 & $3,47-25,74$ & 0,000 \\
\hline $\mathrm{O} / \mathrm{CC}$ normal & 6,97 & $2,14-22,46$ & 0,001 \\
\hline \multirow[t]{2}{*}{$\mathrm{O} / \mathrm{CC}$ aumentada } & 19,35 & $8,19-35,81$ & 0,00 \\
\hline & \multicolumn{3}{|c|}{ Sexo } \\
\hline Mujeres & 1 & & \\
\hline \multirow[t]{2}{*}{ Varones } & 3,07 & $1,8-5,26$ & 0,00 \\
\hline & \multicolumn{3}{|c|}{ Edad } \\
\hline 20-34 años & 1 & & \\
\hline 35-49 años & 1,83 & $0,98-3,43$ & 0,058 \\
\hline 50-64 años & 4,01 & $2,08-7,72$ & 0,000 \\
\hline$\geq 65$ años & 6,11 & $2,4-15,55$ & 0,000 \\
\hline \multicolumn{4}{|l|}{ ALAD } \\
\hline & \multicolumn{3}{|c|}{ Estado nutricional/CC } \\
\hline $\mathrm{N} / \mathrm{CC}$ normal & 1 & & \\
\hline $\mathrm{S} / \mathrm{CC}$ normal & 11,29 & $2,42-52,74$ & 0,002 \\
\hline $\mathrm{S} / \mathrm{CC}$ aumentada & 42,27 & $6,84-261,28$ & 0,000 \\
\hline $\mathrm{O} / \mathrm{CC}$ normal & 24,72 & $4,07-150,1$ & 0,000 \\
\hline \multirow[t]{2}{*}{$\mathrm{O} / \mathrm{CC}$ aumentada } & 174,98 & $37,47-817,68$ & 0,000 \\
\hline & \multicolumn{3}{|c|}{ Sexo } \\
\hline Mujeres & 1 & 1 & \\
\hline \multirow[t]{2}{*}{ Varones } & 9,35 & $4,32-20,23$ & 0,000 \\
\hline & \multicolumn{3}{|c|}{ Edad } \\
\hline 20-34 años & 1 & & \\
\hline 35-49 años & 1,29 & $0,58-2,88$ & 0,53 \\
\hline 50-64 años & 2,51 & $1,11-5,65$ & 0,027 \\
\hline$\geq 65$ años & 1,4 & $0,42-4,63$ & 0,58 \\
\hline
\end{tabular}

N: normal. S: sobrepeso. O: obesidad. CC: circunferencia de cintura. OR p<0,05

\section{Discusión}

El presente trabajo, es uno de los primeros realizados en nuestro país, utilizando el criterio diagnóstico de ALAD para SM. Los resultados expuestos indican que la prevalencia del SM varió según la definición utilizada, con diferencias según sexo y severidad del sobrepeso, reflejándose además que más del $60 \%$ de los adultos de la ciudad de Salta, presentaron $\mathrm{S} / \mathrm{O}$.

Las prevalencias de SM encontradas en Latinoamérica son consistentes entre países y 
dependen de la definición utilizada, de los rangos de edad, de la proporción hombres/ mujeres y del tipo de población (urbana, rural, aborigen). En términos generales puede afirmarse que, una de cada tres o cuatro personas mayores de 20 años cumple criterios para diagnóstico de SM, según cual sea la definición empleada Los estudios en poblaciones argentinas informaron frecuencias que oscilan entre $12,6 \%$ y $26 \%$ para la población general, alcanzando el 53,3\% en los pacientes admitidos en la unidad coronaria .Estudios en poblaciones específicas como, Dean Funes y Oncativo (Córdoba), reportaron que $21 \%$ y $19 \%$ de la población entre 20-70 años presentó SM según definición del ATP III - . El estudio multicéntrico de Coniglio y col. utilizó diferentes definiciones, IDF, Asociación Americana de Cardiología/Instituto Nacional del Corazón, Pulmones y Sangre de EE.UU. y ATP III ,relevando prevalencias de 31, 30 y 26\% respectivamente y mayor en varones . La ciudad de Buenos Aires fue evaluada en el marco del estudio CARMELA, encontrándose una cifra cercana al 17\% entre sujetos de 25-64 años - . En nuestro trabajo la prevalencia de SM en población general fue del 20,9\%, según criterios ALAD y $30,8 \%$ según IDF, mayor en varones (34,2\% ALAD y 41,9\% IDF). En trabajos recientemente publicados, que usaron la definición ALAD, las prevalencias encontradas fueron mayores, Ecuador $43,4 \%$ y Cuba $39,8 \%$.

Con respecto a los componentes del SM, nuestros datos muestran frecuencias más bajas que las observadas por Coniglio y col12:,hipertrigliceridemia 35,9 vs 38,0\%, HDL disminuido 28,2 vs $30,9 \%$ y GB aumentada 11,8 vs $23,8 \%$. Mientras que Luquez y col10 refirieron prevalencias de TG de 34,7\%, HDL 37,4\%y GB aumentada 10,6\%.Ambos trabajos relevan datos de Hipertensión del 56,1 y 35,9\% respectivamente, en nuestra muestra la prevalencia de PAS y PAD elevadas fue de 29,7 y $12,2 \%$ en general, mayor en varones $(44,9$ y $20,0 \%)$.

La obesidad alcanzó en las últimas décadas proporciones epidémicas convirtiéndose en uno de los principales problemas de salud pública en el mundo. En 2014, más de 1900 millones de adultos tenían sobrepeso, de los cuales, más de 600 millones eran obesos .En nuestro país, la $3^{\circ}$ edición de la Encuesta Nacional de Factores de Riesgo, refirió prevalencias de sobrepeso de $37,1 \%$ y obesidad del $20,8 \%$, resultando un $15,6 \%$ mayor que en 2009 y un $42,5 \%$ mayor que en 2005 .Nuestros datos muestran una prevalencia general de sobrepeso del 29,6\% y obesidad 32,2\%, los que revelan un aumento del crecimiento de esta última en relación al sobrepeso, sin diferencias entre sexos. Por otro lado los valores medios de IMC tanto para varones como mujeres se ubicaron en los rangos del sobrepeso $(27,92$ y 28,13$)$.

Los valores de IMC altos se asocian con perfiles de riesgo adversos de morbi-mortalidad. Dentro del concepto de obesidad se han descrito algunos subtipos que complementan la relación dosis-respuesta que existe entre el IMC y sus consecuencias para la salud. Se ha observado la existencia de un fenotipo correspondiente a individuos con peso normal pero metabólicamente obesos es decir, tienen un IMC normal pero presentan las alteraciones típicas de los pacientes obesos: resistencia a la insulina, adiposidad central, bajas cifras de colesterol de HDL y elevación de TG, así como hipertensión arterial. Al mismo tiempo, existen los que se han denominado obesos metabólicamente sanos, que tienen IMC $>30$, pero ninguna de las alteraciones metabólicas típicas de los individuos obesos.Estudios han puesto de manifiesto que la cantidad de tejido adiposo visceral se correlaciona de manera directa, con un perfil de riesgo metabólico, que precede al desarrollo de DM2 y enfermedad cardiovascular. Por lo tanto, aunque es cierto que la obesidad incrementa el riesgo de enfermedades crónicas, parece claro que son los pacientes con obesidad visceral los que forman el subgrupo de individuos con las alteraciones más graves del metabolismo

En el presente trabajo encontramosindividuos con IMC y CC normales que presentan alteraciones metabólicas. La probabilidad de ocurrencia del SM aumentó significativamente, en relación a la presencia conjunta de $\mathrm{S} / \mathrm{O}$ acompañados de $\mathrm{CC}$ aumentada. Por lo tanto 
identificar individuos con mayor riesgo sólo por la medición del peso y la talla no es suficiente, dado que aún con IMC $<$ de $25 \mathrm{Kg} / \mathrm{m}$ existe laposibilidad de presentar aumento del riesgo. Es necesario entonces, identificar a los individuos que poseen mayor tejido adiposo en el compartimiento abdominal.

Los modelos de regresión logística demostraron que la edad, sexo y estado nutricional fueron fuertes predictores de SM en nuestra población, cualquiera sea el criterio diagnóstico empleado. El trabajo de Coniglio y col12 observó sin embargo, que la edad fue un débil predictor, y que los varones tuvieron aproximadamente el doble de riesgo en relación a las mujeres.

Con respecto a los grupos etáreos, Luquez y col10 verificaron un incremento significativo en la prevalencia de SM tanto empleando la definición ATP-III como la OMS. Luego de los 60 años de edad, encomparación con personas de $\leq 30$ años, el riesgo se incrementó 8,8 y 10,1 veces respectivamente. En nuestro estudio el riesgo para el grupoetáreo $\geq 65$ años, según IDF, fue 6,11 veces mayor. Sin embargo usando el criterio ALAD fue 2,51 veces mayor para el grupo de 50-64 años, mientras que el grupo $\geq 65$ años no presentó riesgo significativo.

Conclusiones: La prevalencia de SM en adultos de la ciudad de Salta es altacon el agravante de un marcado $\mathrm{S} / \mathrm{O}$ en la población. Se observaron variaciones importantes según el criterio diagnóstico utilizado, debido a que los puntos de corte de $\mathrm{CC}$ para población latinoamericana son diferentes, por lo que consideramos fundamental incorporar a la evaluación del estado nutricional, la medición de la CCpara la identificación de grupos de riesgo en población.

\section{Bibliografía}

1. García-García Eduardo, De la Llata-Romero Manuel, Kaufer-Horwitz Martha, TusiéLuna María Teresa, Calzada-León Raúl, Vázquez-Velázquez Verónica et al . La obesidad y el síndrome metabólico como problema de salud pública: una reflexión. Salud pública Méx [Internet]. 2008 Dec [cited 2017 Nov 15] ; 50( 6 ): 530-547. Availablefrom: http://www.scielosp.org/scielo.php?script=sci_ar

2. Tercer Encuesta Nacional de Factores de Riesgo. http://www.msal.gob.ar/images/ stories/bes/graficos/0000000544cnt-2015_09_04_encuesta_nacional_factores riesgo.pdf

3. Alberti KG, Eckel RH, Grundy SM, et al. Harmonizing the metabolic syndrome: a joint interim statement of the International Diabetes Federation Task Force on Epidemiology and Prevention; National Heart, Lung, and Blood Institute; American Heart Association; World Heart Federation; International Atherosclerosis Society; and International Association for the Study of Obesity. Circulation 2009; 120:1640-45

4. Rosas Guzmán J., González Chávez A., Aschner P., Bastarrachea R. y col. Epidemiología, Diagnóstico, Control, Prevención y Tratamiento del Síndrome Metabólico en Adultos. Consensos ALAD. 2010. Vol. XVIII. No 1.

5. The IDF consensus worldwide definition of the metabolic syndrome. http://www.idf. org/webdata/docs/IDF_Metasyndrome_definition.pdf..Metasyndrome_definition.pdf

6. BMI Classification. Disponible en: www.apps.who.int/bmi/index jsp

7. Benozzi S, Ordoñez F, Polini N, et al:Insulino resistencia y síndrome metabólico en pacientes con enfermedad coronaria definida por angiografía. MEDICINA (Buenos Aires) 2009;69: 221-28.

8. Luquez H, De Loredo L, Madoery RJ, Luquez H (h),Senestrari D.Síndrome metabólico: prevalencia en dos comunidades de Córdoba, Argentina, de acuerdo con definiciones ATP-III y OMS.RevFedArgCardiol 2005; 34: 80-95.

9. Luquez HA. Síndrome metabólico. Las definiciones actuales y la realidad Argentina. Rev Fed ArgCardiol 2005;34:195-201..

10. Coniglio RI, Nellem J, Gentili R, et al. Estudio IFRALAC 2009: Síndrome metabólico 
en empleados en la Argentina. MEDICINA (Buenos Aires) 2009; 69: 246-52.

11. Schargrodsky H, Hernández R, Marcet-Champagne B, et al.CARMELA Study. Am J Med 2008; 121:5355.

12. Pramparo P, Boissonnet C, Schargrodsky H. Evaluación del riesgo cardiovascular en siete ciudades de Latinoamérica: las principales conclusiones del estudio CARMELA y de los subestudios. Rev. argent. cardiol. [Internet]. 2011 Ago [citado 2017 Mar 30] ; 79( 4 ): 377-382. Disponible en: http://www.scielo. org.ar/scielo.php?script $=$ sci_arttext\&pid $=\mathrm{S} 1850$ 37482011000400014\&lng=es

13. Ortiz-Benavidesb R, Sigüenza-Cruzc W, OrtizBenavidesd A, A neze R, Salazare J, Rojase J y Bermúdeze V .Punto de corte de circunferencia abdominal para el agrupamiento de factores de riesgo metabólico: una propuesta para la población adulta de Cuenca, Ecuador. Rev argent endocrinol metab2016; 53(2):59-66.

14. Bustillo Solano E, Pérez Francisco Y, Brito García A, González Iglesia A, Castañeda Montano D, Santos González M et al .Síndrome metabólico, un problema de salud no diagnosticado. Rev Cubana Endocrinol [Internet]. 2011 Dic [citado 2017 Abr 03] ; 22( 3 ): 167-181. Disponible en: http://scielo. sld.cu/scielo.php?script=sci_arttext\&pid $=\mathrm{S} 1561$ 29532011000300001\&lng=es.

15. Obesidad. Nota descriptiva $\mathrm{N}^{\circ} 311$.Junio de 2016 http://www.who.int/features/factfiles/obesity/es/

16. Tercera Encuesta Nacional de Factores de Riesgo http://www.msal.gob.ar/images/stories/bes/ graficos/0000000544cnt 2015_09_04_encuesta_ nacional_factores_riesgo.pdf.

17. Lizarzaburu Robles JC .Síndrome metabólico: concepto y aplicación práctica. AnFacMed 2013; 74(4):315-20.

18. Ezquerra EA, Castellano Vázquez JM, Barrero AA.Obesidad, síndrome metabólico y diabetes: implicaciones cardiovasculares y actuación terapéutica. RevEspCardiol 2008; 61(7):752-64. 